\title{
Estimating tumour volume in a primary orthotopic mouse model of human pancreatic cancer using rapid acquisition magnetic resonance imaging
}

\author{
Neesha C. Dhani ${ }^{1 *}$, Ines Lohse ${ }^{2}$, Warren D Foltz ${ }^{3}$, Ping-Jiang Cao ${ }^{2}$ and David W Hedley ${ }^{1,2}$ \\ *Correspondence: Neesha.Dhani@uhn.on.ca

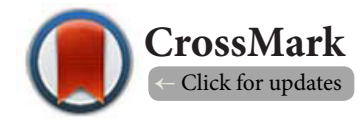 \\ 'Department of Medical Oncology and Hematology, Princess Margaret Cancer Centre, Toronto, Ontario, Canada. \\ 2Ontario Cancer Institute and Campbell Family Cancer Research Institute, Princess Margaret Cancer Centre, University Health Network, \\ Toronto, Ontario. \\ ${ }^{3}$ Department of Radiation Oncology, Princess Margaret Cancer Centre, Canada.
}

\begin{abstract}
Pancreatic cancer has a mortality rate that closely approximates its incidence. This statistic highlights a dismal prognosis that has not improved despite decades of clinical trials. There is optimism that more sophisticated in vivo studies will better predict for clinical efficacy. This includes utilizing more relevant, orthotopic, primary xenograft models and incorporating imaging for tumour burden assessment. There has been hesitation in the routine incorporation of imaging in preclinical studies related primarily to the length and cost of imaging protocols. We hypothesized that rapid acquisition MR scans that balance efficiency with tumour visualization, could be effectively used to provide accurate and timely tumour size estimates. Mice bearing orthotopic primary pancreatic xenografts were study subjects. Animals were imaged on a 7 Tesla preclinical MR scanner, using the most rapid acquisition that provided adequate assessment of tumour size. Two different tumour volume estimates were made -one calculated from 2 dimensions obtained on imaging, and the other a software-based (MIPAV) volumetric analysis. Both were compared with tumour mass (range: 237 to $2575 \mathrm{mg}$ ). Although both calculated tumour volume $\left(\mathrm{V}=1 / 2\left(\mathrm{LxW}^{2}\right)\right)$ and software-based volumetric analysis underestimated tumour size, they correlated well with tumour mass (correlation co-efficient $0.88(\mathrm{p}<0.0001)$ and $0.94(\mathrm{p}<0.0001)$ respectively). Our data demonstrate that rapid acquisition MR protocols can provide accurate tumour volume estimates to allow for longitudinal assessment of growth kinetics. This data has implications for the routine adoption of small animal imaging for preclinical studies using orthotopic pancreatic xenograft models.
\end{abstract}

Keywords: Orthotopic pancreatic tumour, tumour volume, magnetic resonance imaging, small animal imaging

\section{Introduction}

Pancreatic cancer continues to be the $4^{\text {th }}$ leading cause of cancer death despite being only the $10^{\text {th }}$ most frequent cancer diagnosis. This highlights the dismal prognosis of a disease that is almost uniformly fatal with a 5 -year survival of under $5 \%[1,2]$. There remains a critical need to develop novel therapies that can be expeditiously brought forward to the clinic.

Unfortunately, a significant issue compromising drug development in oncology is the poor predictive value of preclinical studies regarding clinical efficacy. Primary xenograft models are directly established from clinical tumour biopsies. These models maintained orthotopically, have demonstrated close alignment with various characteristics of their clinical counterparts, including recapitulation of the tumoral microenvironment and response (or lack thereof) to standard therapies [3-5]. This potentially renders them more relevant models for preclinical studies. In addition, given recent observations that treatment with some anti-cancer therapies such as anti-angiogenic agents, 
may result in primary tumour control while increasing metastatic progression, it is becoming increasing relevant to include metastatic models in preclinical drug evaluation studies $[6,7]$.

The additional complexity introduced into in vivo studies through the use of orthotopic models can be partially resolved with the simultaneous incorporation of more efficient methods of small animal imaging. Still, with a standard animal experiment consisting of 20 to 30 animals, imaging studies need to be both time and cost-effective before they will be routinely assimilated into preclinical investigation. Further, the ability to use imaging studies to assess tumour size in real-time, is preferable to using a protocol that requires additional analysis to obtain equivalent data regarding tumour burden.

The primary goal of this study was to evaluate the ability of a rapid acquisition magnetic resonance (MR)-based imaging strategy to provide accurate tumour volume assessments, thus highlighting the potential of incorporating a similar strategy into large preclinical experiments. We selected tumour mass as the closest estimate of tumour volume, as it has been previously demonstrated to correlate well with pycnometry or water displacement methods of volume estimation, the latter being considered the gold standard [8]. In addition, it is a common endpoint used by our and other laboratories involved in preclinical drug evaluation $[4,9,10]$. Having a method of tumour volume assessment that strongly correlates with tumour mass and can be easily assessed at multiple time-points will allow for a reduction in sample size while maintaining statistical power, highlighting the relevance of this work $[11,12]$.

\section{Methods}

Animal studies were conducted in accordance with animal care regulations of the Ontario Cancer Institute. Since clinical assessment of orthotopic pancreatic tumour size is limited, close attention was paid to signs of animal distress. OCIP51, a primary xenograft model with a rapid and highly variable growth rate (passage time of 8 weeks) was selected for use [13]. Orthotopic xenografts were established in 30 male 4-7 week old SCID mice from the local colony, by surgically implanting $1 \mathrm{~mm}^{3}$ tumour pieces (obtained from a donor tumour) onto the exteriorized tail of the pancreas at laparotomy under inhaled isofluorene anaesthesia, as previously described $[4,9]$.

All 30 animals had an initial scan at 2 weeks post-tumour implantation to evaluate the variability of growth kinetics and for an estimation of baseline tumour volume (as outlined in "MRI protocol" below). From the initial group of 30, 20 with similarly sized tumours were selected to undergo repeat imaging 5 weeks later (i.e., 7 weeks post-tumour implantation). This sample size is representative of the standard preclinical drug experiment conducted in our laboratory. It is also a reasonable number of animals to image during a single imaging session. The animals not imaged at second imaging time-point included 1 animal with metastatic disease and 2 with large tumours (187 and $83 \mathrm{~mm}^{3}$ ) in which there was concern that animals would reach humane endpoints prior to end of study. The remaining
7 animals excluded had very small (11 to $17 \mathrm{~mm}^{3}$ ) tumours at baseline.

The 20 animals selected for repeat MR tumour evaluation were sacrificed after the week 7 imaging study. The same MR protocol was followed for both sets of imaging and is outlined below. At necropsy, primary tumour was carefully resected and weighed to obtain tumour mass.

\section{MRI protocol}

MR imaging was performed on a 7 Tesla preclinical MRI system (Biospec 70/30, Bruker Corporation, Ettlingen, DE) at the SpatioTemporal Targeting and Amplification of Radiation Response (STTARR) preclinical imaging facility, using commerciallyavailable hardware from the MRI system vendor (B-GA12 gradient coil insert, $7.2 \mathrm{~cm}$ inner diameter transmit-only volume coil, and 4-coil murine abdominal phased-array receiver coil). Mice were anaesthetized with $1.8 \%$ isoflurane and oriented such that the orthotopic pancreatic tumour was directly above the phased array coil elements. A pneumatic pillow provided a trace of respiratory motion for gating. An abdominal volume extending from the liver through to the bladder was resolved with a single vertically oriented (axial) T2-weighted Rapid Acquisition with Relaxation Enhancement (RARE) acquisition. The RARE method is applied with an echo time (TE) of $8 \mathrm{~ms}$ and an echo train length of 8 , combining for an effective TE of $32 \mathrm{~ms}$.

Slice coverage and TE determine the minimum repetition time (TR) of the RARE pulse sequence, and this TR must be less than the respiratory cycle duration for fidelity imaging of the mouse abdomen. Selection of slice number of 20 , slice thickness of $1 \mathrm{~mm}$, and TE of $32 \mathrm{~ms}$ provided a suitable trade-off for respiratory cycles of 1.4 seconds or greater (i.e., breathing rates of 42 breaths per minute (bpm) or less) with coverage of the targeted volume. Other sequence parameters included RARE factor of 8, field-of-view of $28 \times 20 \mathrm{~mm}$ at 140x100 matrix for $200 \times 200 \mu \mathrm{m}$ resolution, and $81.5 \mathrm{kHz}$ effective spectral bandwidth. With 4 data averages, the acquisition time was 96,82 , and 70 seconds at respiratory rates of 30,36 , and 42 bpm respectively.

During the imaging study, the slice in which the largest cross-sectional area of tumour was visualized was selected for measurements. Length (L) was defined as the longest diameter, with width (W) the longest diameter perpendicular to and in the same plane as (L) (examples illustrated in Figure 2). Both were recorded and used to derive an approximate tumour volume (designated "calculated tumour volume") using the formula $\mathrm{V}=1 / 2\left(\mathrm{LxW}^{2}\right)$. Although tumour volume calculations using 3 dimensions have been demonstrated to provide the closest estimation of tumour mass, calculations using 2 dimensions have also demonstrated utility and good reproducibility. Given the ease of assessing 2 tumour dimensions on a single axial MR slice, the formula $\mathrm{V}=1 / 2\left(\mathrm{LxW}^{2}\right)$ was selected for these experiments. It is well accepted for use in estimating tumour volume given its demonstrated correlation with tumour mass, the gold standard approximation of tumour volume $[\mathbf{8}, \mathbf{1 4}, \mathbf{1 5}]$. MIPAV (Medical Imaging Processing and Visualization; National Institutes of 
Health, Bethesda, Maryland USA) software was used to obtain a "MRI tumour volume" using manual tumour segmentation as a region of interest or "ROI" across all MR slices. All sections demonstrating some volume of tumour were included. The MIPAV software calculates tumour volume based on total voxel count within the multi-slice ROI, slice thickness and in-plane resolution [16].

\section{Statistical analysis}

Paired t-test was used to compare differences in calculated and MR tumour volumes. Spearman correlation was used to evaluate the relationship between tumour mass and volume and reported as significance of correlation coefficient ( $r$ ).

\section{Results}

A rapidly growing tumour model with highly variable growth kinetics was selected to evaluate the performance of MRI over a range of tumour sizes. At 2 weeks post-implantation, orthotopic tumours were barely detectable by clinical palpation but were easily discernable on MR images acquired over less than 100 sec (Figure 1). Further, length (L) and width (W) tumour dimensions could be obtained in a straightforward manner. There was significant variability in tumour size with calculated tumour volumes (based on $\mathrm{V}=1 / 2\left(\mathrm{LxW}^{2}\right)$ ) at 2 weeks ranging from 11 to $184 \mathrm{~mm}^{3}$ (mean $40 \mathrm{~mm}^{3}$ ).

Twenty animals with calculated tumour volumes ranging from 23 to $70 \mathrm{~mm}^{3}$ (group mean of $40 \mathrm{~mm}^{3}$ ) were selected for serial MR imaging. At repeat imaging 5 weeks later (at

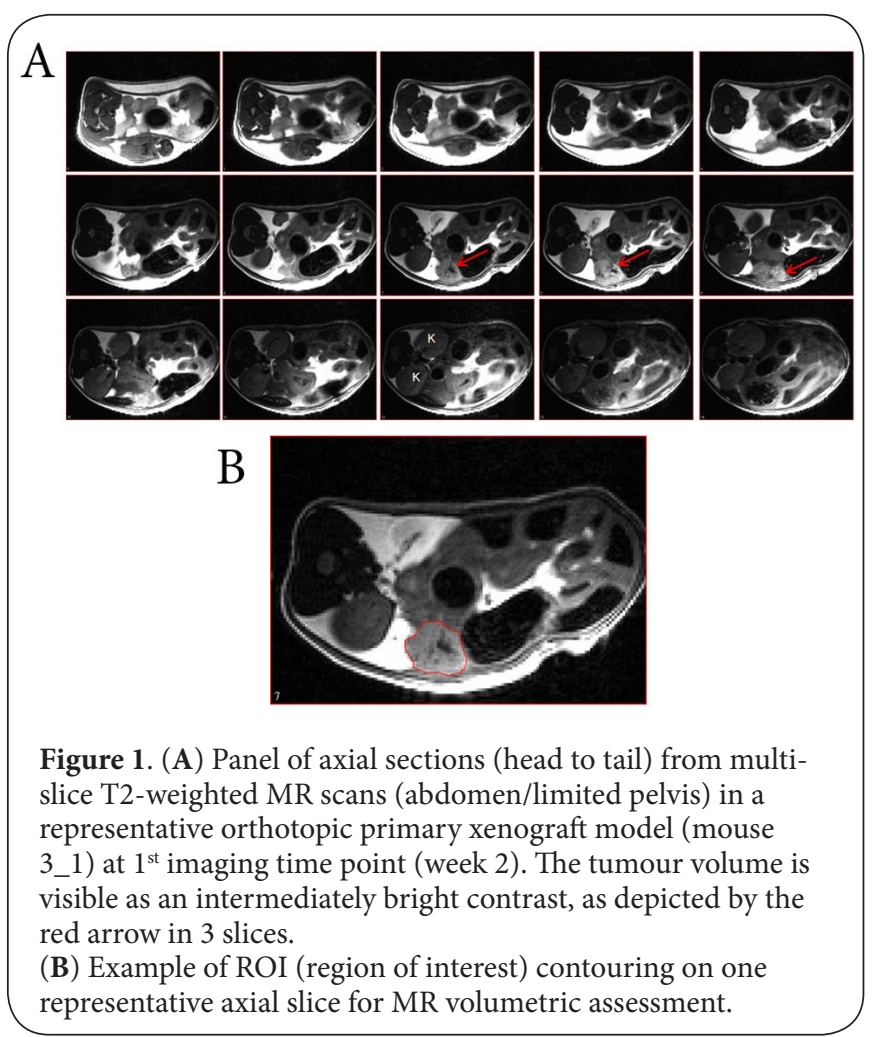

week 7), tumours were once more easy to visualize (Figure 2), and calculated tumour volumes ranged from 88 to $1733 \mathrm{~mm}^{3}$ $\left(\right.$ mean $\left.867 \mathrm{~mm}^{3}\right)$. These volumes corresponded to MR-volumes (obtained by image segmentation using MIPAV) ranging from 136 to $1660 \mathrm{~mm}^{3}$ (mean $982 \mathrm{~mm}^{3}$ ) and tumour mass range of 237 to $2575 \mathrm{mg}$ (mean $1300 \mathrm{mg}$ ). All animals tolerated this range

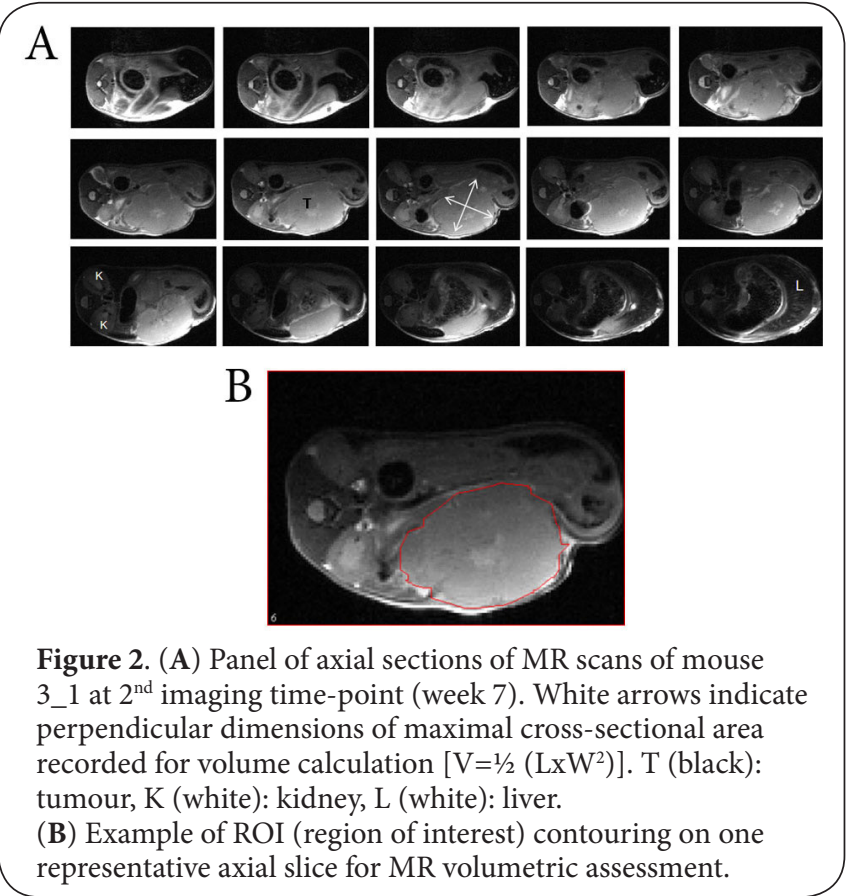

of tumour burden well, exhibiting no signs of morbidity. The rate of change in tumour volume over the 5-week period was also quite variable in keeping with the variable growth kinetics of this model (Figure $\mathbf{3}$ ).

The difference between the two methods of volume calculation was significant with both underestimating tumour volume in comparison with tumour mass. Average error associated with MR and calculated volumes was $319 \mathrm{~mm}^{3}$ (range 100 to 710 $\mathrm{mm}^{3}$ ) and $115 \mathrm{~mm}^{3}$ (range -389 to $281 \mathrm{~mm}^{3}$ ) respectively. Still, in spite of the significant differences in absolute values, there was good correlation between the two methods of tumour volume assessment ( $r=0.96$; CI:0.89-0.98; $\mathrm{p}<0.0001)$. Further, both MRand calculated volumes correlated well with tumour mass - with correlation coefficients of 0.94 (CI:0.85-0.98; $\mathrm{p}<0.0001)$ and 0.88 (CI:0.71-0.95; $\mathrm{p}<0.0001$ ) respectively (Figure 4).

\section{Discussion}

This imaging study in orthotopic primary pancreatic xenograft models was designed to evaluate the efficacy of a rapid acquisition MR study to provide accurate estimates of tumour volume in order to justify incorporation of MR into large-scale preclinical experiments. We demonstrate that an MR acquisition protocol under 100 seconds was sufficient to provide adequate tumour visualization for this purpose. During each mouse scan, the 


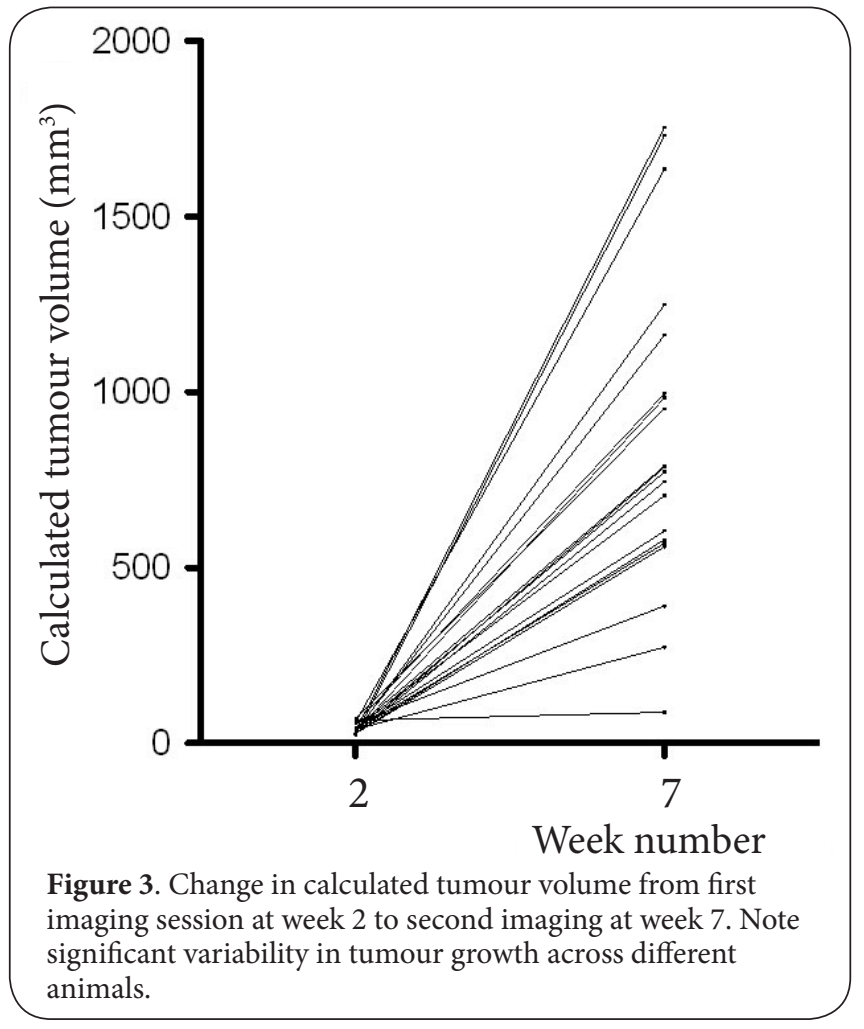

axial MR slice in which the maximal cross-sectional area of tumour was visualized was selected, and tumour dimensions representing (L) and (W) were recorded. These were used to calculate tumour volume in real-time, using the formula for the volume of an ellipsoid, $\mathrm{V}=1 / 2\left(\mathrm{LxH}^{2}\right)$ which has demonstrated good correlation with tumour mass and is considered a gold standard for volumetric calculations using 2 tumour dimensions. This "calculated tumour volume" was closely aligned with (correlation coefficient 0.96 ) the "MR tumour volume" obtained by standard software-based volumetric analysis involving sliceby-slice image segmentation. The MR volume analysis took on average an additional $10 \mathrm{~min}$ per mouse, and was completed after actual mouse imaging. Although the latter method provided a tumour volume estimate that better correlated with tumour mass (correlation coefficient 0.94 vs 0.88 ), the former was more timely, less labour intensive, and easier to conduct in real-time. Reassuringly, we observed that the performance of calculated tumour volume (based on 2 dimensions obtained from MR) agreed with reports in the literature in subcutaneous xenografts [15].

We and others have demonstrated that primary pancreatic xenograft models grown orthotopically, closely align with clinical pancreatic cancers with respect to recapitulation of the tumoral microenvironment, tendency to local invasion and metastasis and response to standard treatments [3-5]. Given these parallels, preclinical evaluation of novel therapeutic targets utilizing these models is hypothesized to have a higher positive predictive value for clinical efficacy $[17,18]$. However, the use of orthotopic pancreatic models introduces additional levels of complexity to in vivo drug experiments. Since pancreatic tumours are located deep in the abdomen they are difficult to follow longitudinally by clinical palpation and are impossible to monitor by calliper measurements. Although final tumour weight can be effectively used as an endpoint outcome measure, any uncertainty regarding baseline tumour measurements may introduce unwanted variability to an experiment. This is not an insignificant problem since the cellular heterogeneity that make primary models attractive as models also translates into variability with respect to growth rates as we demonstrate here with the heterogeneity in tumour size at baseline evaluation and in change in tumour volume over time. Further, there is a high probability that some animals may be tolerating a high
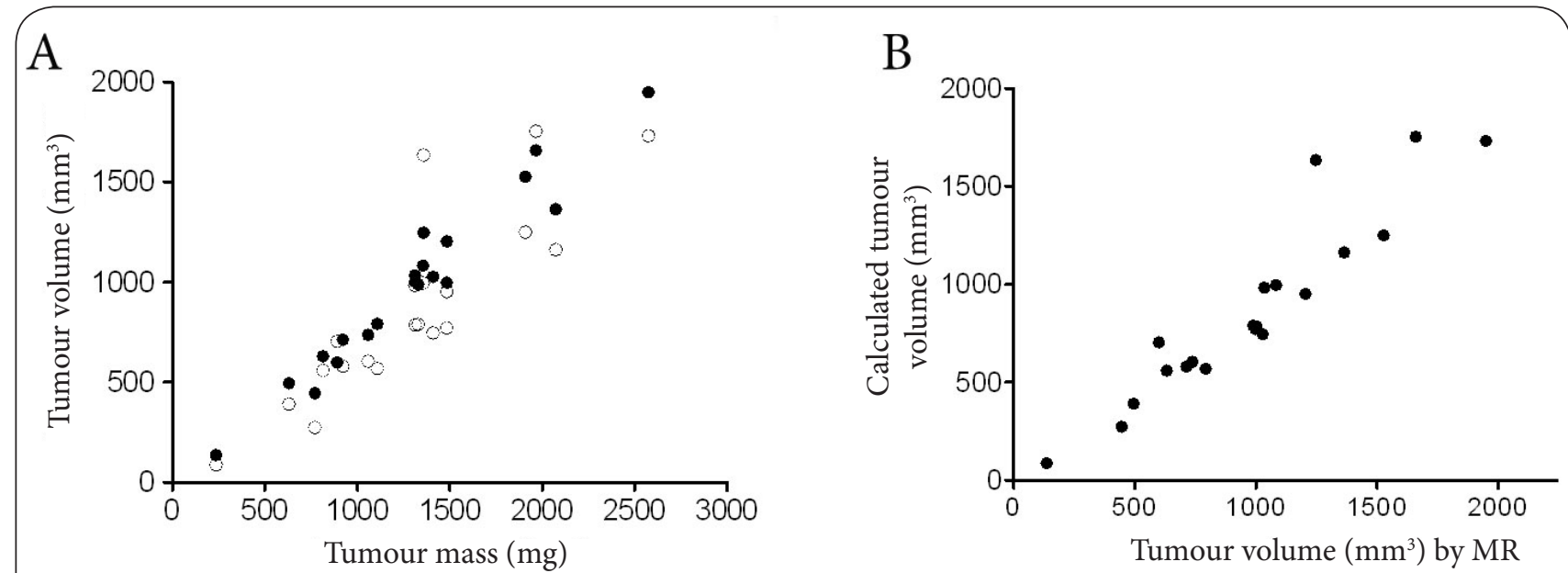

Figure 4. Scatterplot graphs demonstrating correlation between (A) tumour mass and estimated tumour volume [volume by MR volumetric analysis in black circles $(\mathrm{r}=0.94 ; \mathrm{p}<0.0001)$ and calculated from two dimensions of maximal cross-sectional area in open circles $(\mathrm{r}=0.88 ; \mathrm{p}<0.0001)]$ and $(\mathbf{B})$ both methods of tumour volume (in $\left.\mathrm{mm}^{3}\right)$ assessment $(\mathrm{r}=0.96 ; \mathrm{p}<0.0001)$. 
burden of disease at endpoint despite no evidence of morbidity. A method of non-invasive tumour assessment, which can be rapidly applied to a large group of animals in a cost effective manner, would improve the statistical power of experiments through increased accounting for inherent variability.

It is also important to note that the short time for acquisition of imaging studies limits anaesthesia exposure to the animals. In addition, the lack of requirement of an exogenous contrast agent also simplifies both the imaging protocol itself as well as potential trauma to the animal. Since all animals in an experiment (i.e., controls as well as those undergoing treatment) would be imaged any effects related to the stress of additional handling or imaging procedure itself would be applied uniformly to all animals and should not influence outcomes. We have applied rapid acquisition imaging (using both $\mathrm{CT}$ and $\mathrm{MR}$ ) longitudinally in animal experiments and noted no detrimental effects.

There have been prior reports demonstrating the utility of non-invasive imaging modalities (MR and ultrasound) for accurate monitoring of tumour size $[\mathbf{1 1 , 1 2}]$. These two studies compared imaging based volumetric assessment with volume calculations derived from calliper measurements in subcutaneous xenograft tumours. Both demonstrated superior accuracy of imaging techniques but the generalizability of this data to intraabdominal tumours was unclear. A more recent study confirmed the validity of MR to monitor orthotopic pancreatic tumours. However, these scans were lengthy (15 min on average) which may be both time and cost prohibitive for large, multi-cohort animal studies [19]. Alternate imaging techniques such as bioluminescence have also been investigated for longitudinal assessment of tumour progression in animal models given it allows for rapid and inexpensive screening based on detection of luciferase production. It however provides limited information regarding tumour volumetry given the poor resolution of projection images. Our experience has been that users at our institution prefer to combine bioluminescence imaging with higher resolution MRI with the latter providing information on tumour dimensions.. Further, bioluminescence can only be used in transfectable models and has limited utility in the patient-derived xenograft models that we utilize for preclinical drug testing.

As mentioned previously, we selected tumour mass as the gold standard against which to evaluate the two methods of volume assessment given the prior demonstration that tumour mass tracks well with volume by pyncometry or volume displacement. In addition, the same study also demonstrated good correlation between tumour volume calculated from 2 (calliper-made) dimensions with both tumour mass and pycnometry [8]. As a result we decided to use 2 dimensions for our tumour volume calculations. We were cognizant of other reports where formulae incorporating 3 tumour dimensions ( $\mathrm{L}, \mathrm{W}, \mathrm{H})$ appeared to correlate best with actual tumour volume [15]. However, these studies were based on subcutaneous xenograft tumours, easily accessible in three dimensions. Work in liver tumours, in which only 2 dimensions were easily accessible, demonstrated good reliability of the ellipsoid volume formula $\mathrm{V}=1 / 2\left(\mathrm{LXW}^{2}\right)$ to estimate tumour volume, sufficient to render it acceptable as a standard in the field for tumour volume calculations [14]. Also noteworthy is the finding that attempts to obtain a height $(\mathrm{H})$ dimension often introduces the most significant variability (and therefore greatest error) in tumour volume estimates $[\mathbf{2 0 , 2 1}$. Further, in subcutaneous models, calliper based measurements (applied to calculation $\left.\mathrm{V}=1 / 2\left(\mathrm{LXW}^{2}\right)\right)$ have been found to correlate well with volume by water displacement [12]. From a practical viewpoint and in the context of this work, we felt that a calculation using 2 dimensions ( $\mathrm{L}$ and $\mathrm{W}$ ) was preferable, as both of these can be obtained from a single axial MR slice in a reproducible manner.

\section{Conclusions}

We demonstrate here that limited MR studies can be practically incorporated into large animal experiments to provide an estimate of tumour volume (through volumetric analysis) that correlates well with tumour mass $(\mathrm{r}=0.94 ; \mathrm{p}<0.0001)$ across a wide range of tumour sizes ( 237 to $2575 \mathrm{mg}$ ). In addition, perpendicular diameters taken from the maximal axial cross-sectional area of tumour can be used in tumour volume calculations to provide a reasonably good estimate of tumour volume.

\section{List of abbreviations}

L: (tumour) length; defined as longest diameter of maximal crosssectional tumour area

MIPAV: Medical Imaging Processing and Visualization

MRI: Magnetic resonance imaging

RARE: Rapid acquisition with relaxation enhancement

ROI: Region of Interest

V: Volume

$\mathrm{W}$ : (tumour) width; defined as longest diameter perpendicular to "length"

\section{Competing interests}

The authors declare that they have no competing interests.

\section{Authors' contributions}

ND, IL, DWH: planning of experiments, preparation of manuscript, ND: statistical analysis, PJ, IL: animal model, ND, WDF: MRI, MRI analysis.

\section{Acknowledgement}

This work was supported in part by research funding from the Ontario Institute of Cancer Research. We would also like to acknowledge the Spatio-Temporal Targeting and Amplification of Radiation Response (STTARR) program and its affiliated funding agencies. Finally, we thank Drs Tuula Kalliomaki and Joerg Schwock for helpful discussions during the preparation of this manuscript.

\section{Publication history}

Received: 19 September 2014 Revised: 05 November 2014 Accepted: 18 November 2014 Published: 29 November 2014

\section{References}

1. Canel, M., Secades, P., Garzon-Arango, M., Allonca, E., Suarez, C., Serrels, A., Frame, M., Brunton, V. and Chiara, M. D. (2008). 
Dhani et al. Journal of Cancer Therapeutics \& Research 2014,

Involvement of focal adhesion kinase in cellular invasion of head and neck squamous cell carcinomas via regulation of MMP-2 expression. Br J Cancer, 98, 1274-84. |

2. Yang, M. H. and Wu, K. J. (2008). TWIST activation by hypoxia inducible factor-1 (HIF-1): implications in metastasis and development. Cell Cycle, 7, 2090-6.

3. Jin, K., Teng, L., Shen, Y., He, K., Xu, Z. and Li, G. (2010). Patientderived human tumour tissue xenografts in immunodeficient mice: a systematic review. Clin Transl Oncol, 12, 473-80.

4. Ng, S. S., Tsao, M. S., Nicklee, T. and Hedley, D. W. (2002). Effects of the epidermal growth factor receptor inhibitor OSI-774, Tarceva, on downstream signaling pathways and apoptosis in human pancreatic adenocarcinoma. Mol Cancer Ther, 1, 777-83.

5. Rubio-Viqueira, B., Jimeno, A., Cusatis, G., Zhang, X., IacobuzioDonahue, C., Karikari, C., Shi, C., Danenberg, K., Danenberg, P. V. and Kuramochi, H. et al. (2006). An in vivo platform for translational drug development in pancreatic cancer. Clin Cancer Res, 12, 4652-61.

6. Ebos, J. M., Lee, C. R., Cruz-Munoz, W., Bjarnason, G. A., Christensen, J. G. and Kerbel, R. S. (2009). Accelerated metastasis after short-term treatment with a potent inhibitor of tumor angiogenesis. Cancer Cell, 15, 232-9.

7. Loges, S., Mazzone, M., Hohensinner, P. and Carmeliet, P. (2009). Silencing or fueling metastasis with VEGF inhibitors: antiangiogenesis revisited. Cancer Cell, 15, 167-70.

8. Euhus, D. M., Hudd, C., LaRegina, M. C. and Johnson, F. E. (1986). Tumor measurement in the nude mouse. J Surg Oncol, 31, 229-34.

9. Cao, P., Maira, SM., Garcia-Echeverria, C. and Hedley, DW. (2009). Activity of a novel, dual PI3-kinase/mTor inhibitor NVPBEZ235 against primary human pancreatic cancers grown as orthotopic xenografts. Br J Cancer, 100, 1267-76.

10. Yau, C. Y., Wheeler, J. J., Sutton, K. L. and Hedley, D. W. (2005). Inhibition of integrin-linked kinase by a selective small molecule inhibitor, QLT0254, inhibits the PI3K/PKB/mTOR, Stat3, and FKHR pathways and tumor growth, and enhances gemcitabineinduced apoptosis in human orthotopic primary pancreatic cancer xenografts. Cancer Res, 65, 1497-504.

11. Ayers, GD., McKinley, ET., Zhao, P., Fritz, JM., Metry, RE., Deal, BC., Adlerz, KM., Coffey, RJ. And Manning, HC. (2010). Volume of preclinical xenograft tumors is more accurately assessed by ultrasound imaging than manual caliper measurements. $J$ Ultrasound Med, 29, 891-901.

12. Cornelissen, B., Kersemans, V., Jans, L., Staelens, L., Oltenfreiter, R., Thonissen, T., Achten, E. and Slegers G. (2005). Comparison between $1 \mathrm{~T}$ MRI and non-MRI based volumetry in inoculated tumours in mice. Br J Radiol, 78, 338-42.

13.Lohse, I., Lourenco, C., Ibrahimov, E., Pintilie, M., Tsao, M. S. and Hedley, D. W. (2014). Assessment of hypoxia in the stroma of patient-derived pancreatic tumor xenografts. Cancers (Basel), 6, 459-71.

14. Carlsson, G., Gullberg, B. and Hafstrom, L. (1983). Estimation of liver tumor volume using different formulas - an experimental study in rats. J Cancer Res Clin Oncol, 105, 20-3.

15. Tomayko, M. M. and Reynolds, C. P. (1989). Determination of subcutaneous tumor size in athymic (nude) mice. Cancer Chemother Pharmacol, 24, 148-54.

16. McAuliffe, M., Lalonde, F., MCGarry, D., Gandler, W., Csaky, K. and Trus, B. (2001). Medical Imaging Processing Analysis \& Visualization in Clinical Research. IEEE Computer-Based Medical Systems, 381-6.
17.Schuh, J. C. (2004). Trials, tribulations, and trends in tumor modeling in mice. Toxicol Pathol, 32 Suppl 1, 53-66.

18. Troiani, T., Schettino, C., Martinelli, E., Morgillo, F., Tortora, G. and Ciardiello, F. (2008). The use of xenograft models for the selection of cancer treatments with the EGFR as an example. Crit Rev Oncol Hematol, 65, 200-11.

19. Partecke, I. L., Kaeding, A., Sendler, M., Albers, N., Kuhn, J. P., Speerforck, S., Roese, S., Seubert, F., Diedrich, S., Kuehn, S., Weiss, U. F., Mayerle, J., Lerch, M. M., Hadlich, S., Hosten, N., Heidecke, C. D., Puls, R. and von Bernstorff, W. (2011). In vivo imaging of pancreatic tumours and liver metastases using 7 Tesla MRI in a murine orthotopic pancreatic cancer model and a liver metastases model. BMC Cancer, 11, 40.

20.Dethlefsen, L. A., Prewitt, J. M. and Mendelsohn, M. L. (1968). Analysis of tumor growth curves. J Natl Cancer Inst, 40, 389-405.

21.Feldman, J., Goldwasser, R., Mark, S., Schwartz, J. and Orion, I. (2009). A Mathematical Model for Tumor Volume Evaluation using Two-Dimensions. Journal of Applied Quantitative Methods, $4,455-65$.

Citation:

Dhani NC, Lohse I, Foltz WD, Cao PJ and Hedley DW. Estimating tumour volume in a primary orthotopic mouse model of human pancreatic cancer using rapid acquisition magnetic resonance imaging. $J$ Cancer Ther Res. 2014; 3:9.

http://dx.doi.org/10.7243/2049-7962-3-9 